\title{
Peristaltic transport through eccentric cylinders: Mathematical model
}

\author{
Kh.S. Mekheimer ${ }^{\mathrm{a}, \mathrm{b}, *}$, Y. Abd elmaboud ${ }^{\mathrm{c}, \mathrm{d}}$ and A.I. Abdellateef $\mathrm{f}^{\mathrm{e}}$ \\ ${ }^{a}$ Mathematics \& Statistic Department, Faculty of Science, Taif University, Hawia(888) Taif, Saudi Arabia \\ ${ }^{\mathrm{b}}$ Mathematical Department, Faculty of Science (Men), Al-Azhar University, Nasr City, Cairo, Egypt \\ ${ }^{\mathrm{c}}$ Mathematics Department, Faculty of Science, Al-Azhar University(Assiut Branch), Assiut, Egypt \\ ${ }^{\mathrm{d}}$ Mathematics Department, Faculty of Science and Arts, Khulais, King Abdulaziz University (KAU), Saudi Arabia \\ ${ }^{\mathrm{e}}$ Basic science Department, Higher Institute of Engineering, Shorouk City, Cairo, Egypt
}

\begin{abstract}
This paper discusses the effect of peristaltic transport on the fluid flow in the gap between two eccentric tubes (eccentric-annulus flows). The inner tube is uniform, rigid, while the outer tube has a sinusoidal wave traveling down its wall. The flow analysis has been developed for low Reynolds number and long wave length approximation. The velocity and the pressure gradient have been obtained in terms of the dimensionless flow rate $\bar{Q}$, time $t$, azimuthal coordinate $\theta$ and eccentricity parameter $\epsilon$ (the parameter that controls of the eccentricity of the inner tube position). The results show that there is a significant deference between eccentric and concentric annulus flows.
\end{abstract}

Keywords: Peristaltic flow, endoscope, eccentric-annulus flows

\section{Introduction}

Endoscope and catheter are very important tools for medical diagnosis and they have many clinical applications. The endoscope now is a very important tool used for determining real reasons responsible for many problems in the human organs in which the fluid are transported by peristaltic pumping such as, stomach, small intestine, etc. Also from fluid dynamic point of view, there is no difference between an endoscope and catheter. In medicine, a catheter is a tube that can be inserted into a body cavity, duct or vessel. Catheters thereby allow drainage or injection of fluids. The process of inserting a catheter is catheterization.

*Corresponding author: Kh.S. Mekheimer, Mathematics \& Statistic Department, Faculty of Science, Taif University, Hawia(888) Taif, Saudi Arabia; Mathematical Department, Faculty of Science (Men), Al-Azhar University, Nasr City, Cairo, Egypt. E-mail: Kh_Mekheimer@yahoo.com.
Also, the injection of fluid into the body using a needle or syringe is an important application of fluid dynamics. In the surgical process known as thread injection a surgeon not only injects fluid into the body, but also various medical implants. A particular application of chemical thread injection is in lip augmentation found in most plastic surgery industries.

Peristaltic transport is a form of fluid transport that occurs when a progressive wave of area contraction or expansion propagates along the length of distensible duct. Peristalsis is an inherent property of many biological systems having smooth muscle tubes which transports biofluids by its propulsive movements and is found in the transport of urine from kidney to the bladder, the movement of chyme in the gastro-intestinal tract, intra-uterine fluid motion, vasomotion of the small blood vessels and in many other glandular ducts.

In recent years peristaltic transport through a concentric annulus has received much attention. There are several investigations to study the effect of an 


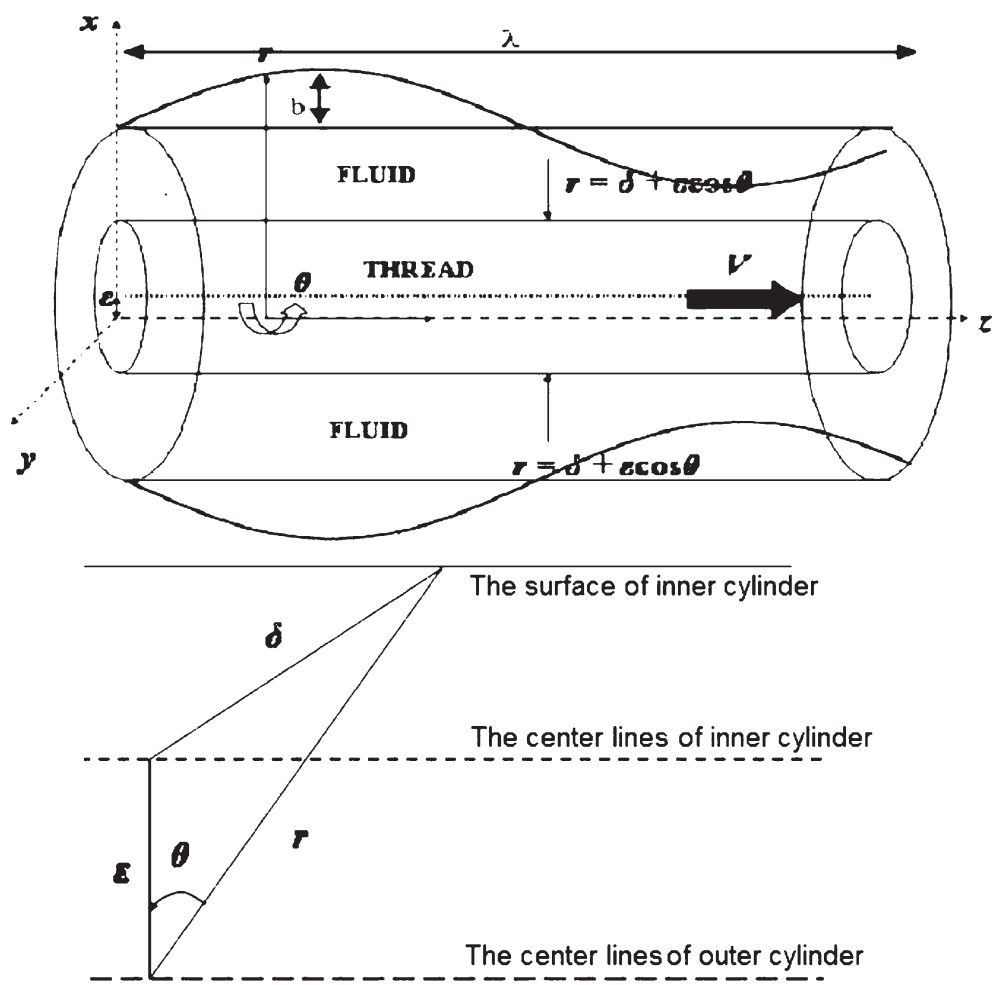

Fig. 1. The simplified model geometry of the problem.

endoscope on peristaltic motion for a Newtonian and non-Newtonian fluids [1-13].

The eccentric annulus (without peristaltic) is somewhat more difficult to treat. Few studies take into consideration the effect of the eccentricity property [14-18].

No attempts have been made yet to discuss peristaltic flow through the eccentric annulus. Therefore the main purpose of the present paper is to discuss the effect of peristaltic transport on the fluid flow in the gap between two eccentric tubes, the outer tube have a sinusoidal wave traveling down its wall and the inner one is rigid. A motivation of the present analysis is the hope that such a problem will be applicable in many clinical applications. This investigation give a better understanding for the speed of injection and the fluid flow characteristics within the syringe. Also, the injection can be carried out more proficiently and the pain of the patient can be reduced.

\section{Formulation of the problem}

Consider a flow of an incompressible Newtonian fluid through eccentric tubes. The inner tube is rigid (endoscope or catheter) and the outer have a sinusoidal wave traveling down its wall. The radius of the inner tube is $\delta^{\prime}$ but we need to reference the fluid motion to the center of the outer tube. The center of the inner tube is now at position $z^{\prime}=\epsilon^{\prime}, y^{\prime}=0$ where $y^{\prime}$ and $z^{\prime}$ are coordinates in the cross-section of the pipe as shown in Fig. 1. Then the boundary of the inner tube is described to order $\epsilon^{\prime}$ by $r_{1}^{\prime}=\delta^{\prime}+\epsilon^{\prime} \cos \left[\theta^{\prime}\right]$. (obtained by using the cosine rule) where $\left(\epsilon^{\prime}<<\delta^{\prime}\right)$ is the parameter that controls the eccentricity of the inner tube position. The geometry of the walls surface is described in Fig.1, the equations for the radii are

$$
\begin{aligned}
& r_{1}^{\prime}=\delta^{\prime}+\epsilon^{\prime} \cos \left[\theta^{\prime}\right], \\
& r_{2}^{\prime}=a^{\prime}+b^{\prime} \cos \left[\frac{2 \pi}{\lambda}\left(z^{\prime}-c t^{\prime}\right)\right],
\end{aligned}
$$

where $\delta^{\prime}, a^{\prime}$ are the radius of the inner and the outer tubes, $b^{\prime}$ is the amplitude of the wave, $\lambda^{\prime}$ is the wavelength, $c^{\prime}$ is the propagation velocity and $t^{\prime}$ is the time. The problem has been studied in cylindrical coordinate system $\left(r^{\prime}, \theta^{\prime}, z^{\prime}\right)$, radial, azimuthal and axial coordinates respectively. 
The equations governing the problem are:

$$
\begin{gathered}
\frac{\partial u^{\prime}}{\partial z^{\prime}}+\frac{\partial v^{\prime}}{\partial r^{\prime}}+\frac{v^{\prime}}{r^{\prime}}+\frac{1}{r^{\prime}} \frac{\partial w^{\prime}}{\partial \theta^{\prime}}=0 \\
\rho\left[\frac{\partial u^{\prime}}{\partial t^{\prime}}+u^{\prime} \frac{\partial u^{\prime}}{\partial z^{\prime}}+v^{\prime} \frac{\partial u^{\prime}}{\partial r^{\prime}}+\frac{w^{\prime}}{r^{\prime}} \frac{\partial u^{\prime}}{\partial \theta^{\prime}}\right] \\
=-\frac{\partial p^{\prime}}{\partial z^{\prime}}+\mu\left[\frac{\partial^{2} u^{\prime}}{\partial z^{\prime 2}}+\frac{\partial^{2} u^{\prime}}{\partial r^{\prime 2}}+\frac{1}{r^{\prime}} \frac{\partial u^{\prime}}{\partial r^{\prime}}\right. \\
\left.+\frac{1}{r^{\prime 2}} \frac{\partial^{2} u^{\prime}}{\partial \theta^{\prime 2}}\right]
\end{gathered}
$$

$$
\begin{gathered}
\rho\left[\frac{\partial v^{\prime}}{\partial t^{\prime}}+u^{\prime} \frac{\partial v^{\prime}}{\partial z^{\prime}}+v^{\prime} \frac{\partial v^{\prime}}{\partial r^{\prime}}+\frac{w^{\prime}}{r^{\prime}} \frac{\partial v^{\prime}}{\partial \theta^{\prime}}-\frac{w^{\prime 2}}{r^{\prime}}\right] \\
=-\frac{\partial p^{\prime}}{\partial r^{\prime}}+\mu\left[\frac{\partial^{2} v^{\prime}}{\partial z^{\prime 2}}+\frac{\partial^{2} v^{\prime}}{\partial r^{\prime 2}}+\frac{1}{r^{\prime}} \frac{\partial v^{\prime}}{\partial r^{\prime}}\right. \\
\left.+\frac{1}{r^{\prime 2}} \frac{\partial^{2} v^{\prime}}{\partial \theta^{\prime 2}}-\frac{v^{\prime}}{r^{\prime 2}}-\frac{2}{r^{\prime 2}} \frac{\partial^{2} w^{\prime}}{\partial \theta^{\prime 2}}\right]
\end{gathered}
$$

$$
\begin{gathered}
\rho\left[\frac{\partial w^{\prime}}{\partial t^{\prime}}+u^{\prime} \frac{\partial w^{\prime}}{\partial z^{\prime}}+v^{\prime} \frac{\partial w^{\prime}}{\partial r^{\prime}}+\frac{w^{\prime}}{r^{\prime}} \frac{\partial w^{\prime}}{\partial \theta^{\prime}}+\frac{w^{\prime} v^{\prime}}{r^{\prime}}\right] \\
=-\frac{\partial p^{\prime}}{\partial \theta^{\prime}}+\mu\left[\frac{\partial^{2} w^{\prime}}{\partial z^{\prime 2}}+\frac{\partial^{2} w^{\prime}}{\partial r^{\prime 2}}+\frac{1}{r^{\prime}} \frac{\partial w^{\prime}}{\partial r^{\prime}}+\right. \\
\left.\frac{1}{r^{\prime 2}} \frac{\partial^{2} w^{\prime}}{\partial \theta^{\prime 2}}-\frac{w^{\prime}}{r^{\prime 2}}+\frac{2}{r^{\prime 2}} \frac{\partial v^{\prime}}{\partial \theta^{\prime}}\right]
\end{gathered}
$$

Where $v^{\prime}, w^{\prime}, u^{\prime}$ are the velocity components in $r^{\prime}$, $\theta^{\prime}$ and $z^{\prime}$-directions respectively, $\rho$ is the density, $p^{\prime}$ is the pressure and $\mu$ is the viscosity. The boundary conditions are:

$$
\begin{aligned}
& u^{\prime}=0, \quad v^{\prime}=\frac{\partial r_{2}^{\prime}}{\partial t}, \quad w^{\prime}=0 \quad \text { at } \quad r^{\prime}=r_{2}^{\prime}, \\
& u^{\prime}=V^{\prime}, \quad v^{\prime}=0, \quad w^{\prime}=0, \quad \text { at } \quad r^{\prime}=r_{1}^{\prime},
\end{aligned}
$$

where $V^{\prime}$ is the velocity of the inner tube. We will assume that the velocity vector in the form $\left(0,0, u^{\prime}\right)$ because the effective velocity component only is the axial velocity. We introduce the following nondimensional variables and parameters

$$
\begin{aligned}
p & =\frac{a^{2}}{\mu c \lambda} p^{\prime}, u=\frac{u^{\prime}}{c}, \theta=\theta^{\prime}, \mathrm{t}=\frac{\mathrm{c}}{\lambda} \mathrm{t}^{\prime}, \\
\operatorname{Re} & =\frac{\rho c a}{\mu}, r_{1}=\frac{r_{1}^{\prime}}{a}=\frac{\delta^{\prime}}{a}+\frac{\epsilon^{\prime}}{a} \cos \left(\theta^{\prime}\right) \phi=\frac{b}{a}, \\
\delta & =\frac{\delta^{\prime}}{a}, \epsilon=\frac{\epsilon^{\prime}}{a}, \delta_{0}=\frac{a}{\lambda}, \mathrm{z}=\frac{\mathrm{z}^{\prime}}{\lambda}, \\
r_{2} & =\frac{r_{2}^{\prime}}{a}=1+\frac{b^{\prime}}{a} \cos (2 \pi(z-t)),
\end{aligned}
$$

where $\phi$ is the amplitude ratio, $R e$ is the Reynolds number, $\delta_{0}$ is the dimensionless wave number, $\epsilon$ is eccentricity parameter. After using the above assumption, the equations of motion in the dimensionless form become

$$
\frac{\partial u}{\partial z}=0
$$

$$
\begin{aligned}
& \operatorname{Re} \delta_{0}\left[\frac{\partial \mathrm{u}}{\partial \mathrm{t}}+\mathrm{u} \frac{\partial \mathrm{u}}{\partial \mathrm{z}}\right]=-\frac{\partial p}{\partial z}+\left[\delta_{0}^{2} \frac{\partial^{2} u}{\partial z^{2}}+\frac{\partial^{2} u}{\partial r^{2}}\right. \\
&\left.+\frac{1}{r} \frac{\partial u}{\partial r}+\frac{1}{r^{2}} \frac{\partial^{2} u}{\partial \theta^{2}}\right] \\
& \frac{\partial p}{\partial r}=0 \\
& \frac{\partial p}{\partial \theta}=0
\end{aligned}
$$

Equations $(2.10,2.11)$ shows that $p$ is not a function of $r$ and $\theta$. Using the long wavelength approximation and dropping terms of order $\delta_{0}$ and higher, it follows from Equations $(2.8,2.11)$ that the appropriate equation describing the flow is

$$
\frac{d p}{d z}=\left[\frac{\partial^{2} u}{\partial r^{2}}+\frac{1}{r} \frac{\partial u}{\partial r}+\frac{1}{r^{2}} \frac{\partial^{2} u}{\partial \theta^{2}}\right] .
$$

The basic flow is assumed to be unsteady and unidirectional so a solution of Equation (2.15) is written in the form $u(r(z, t), \theta)=u_{0}(r)+\epsilon \cos (\theta) u_{1}(r)+\ldots$, the two variable $z, t$ will appear from the boundary conditions. Substituted into Equation (2.15) and collect the like power then the equations for $u_{0}$ and $u_{1}$ are found to be

$$
\begin{aligned}
& \frac{\partial^{2} u_{0}}{\partial r^{2}}+\frac{1}{r} \frac{\partial u_{0}}{\partial r}=\frac{d p}{d z} \\
& \frac{\partial^{2} u_{1}}{\partial r^{2}}+\frac{1}{r} \frac{\partial u_{1}}{\partial r}-\frac{1}{r^{2}} u_{1}=0
\end{aligned}
$$


The boundary conditions are obtained from the manipulation of the viscous no-slip conditions at the boundary of the wall of the outer and inner tubes,

$$
\begin{aligned}
& u=0, \text { on } \quad r=r_{2}=1+\phi \cos (2 \pi(z-t)), \\
& u=V, \text { on } \quad r=r_{1}=\delta+\epsilon \cos (\theta) .
\end{aligned}
$$

As $\frac{d p}{d z}=4, \phi=0, r_{2}=1$ (No peristaltic pumping) our results reduce to that given by Labadin and Walton [18] and for $\epsilon=0$ the results reduce to concentric case $u_{0}$, that represented by Mekheimer [7].

The instantaneous volume flow rate $Q(z, t)$ is given by

$$
\begin{aligned}
Q(z, t) & =2 \pi \int_{\mathrm{r} 1}^{\mathrm{r} 2} r u d r, Q / 2 \pi=\frac{1}{48\left(r_{2}-\delta\right)\left(r_{2}+\delta\right) \log \left[\frac{\delta}{r_{2}}\right]} \frac{d p}{d z} \\
& \left(3 ( r _ { 2 } - \delta ) ( r _ { 2 } + \delta ) \left(-\left(r_{2}-\delta\right)\left(r_{2}+\delta\right)\left(-r_{1}^{2}+r_{2}^{2}+2 r_{1}^{2} \log \left[\frac{r_{1}}{r_{2}}\right]-2 r_{2}^{2} \log \left[\frac{r_{2}}{r_{2}}\right]\right)\right.\right. \\
& \left.+\left(-r_{1}^{4}+r_{2}^{4}+2 r_{2}^{2}\left(r_{1}-r_{2}\right)\left(r_{1}+r_{2}\right)\right) \log \left[\frac{\delta}{r_{2}}\right]\right)+ \\
& \left.4\left(-r_{1}^{3}+3 r_{2}^{2}\left(r_{1}-r_{2}\right)+r_{2}^{3}\right) \epsilon \operatorname{Cos}[\theta]\left(r_{2}^{2}-\delta^{2}+2 \delta^{2} \log \left[\frac{\delta}{r_{2}}\right]\right)\right) \\
& \frac{V\left(4\left(-r_{1}^{3}+3 r_{2}^{2}\left(r_{1}-r_{2}\right)+r_{2}^{3}\right) \epsilon \operatorname{Cos}[\theta]-3\left(r_{2}^{2}-\delta^{2}\right)\left(-r_{1}^{2}+r_{2}^{2}+2 r_{1}^{2} \log \left[\frac{r_{1}}{r_{2}}\right]-2 r_{2}^{2} \log \left[\frac{r_{2}}{\mathrm{r}_{2}}\right]\right)\right)}{12\left(r_{2}^{2}-\delta^{2}\right) \log \left[\frac{\delta}{r_{2}}\right]}
\end{aligned}
$$

By using Taylor series, the viscous no-slip conditions are:

$$
\begin{aligned}
& u_{0}\left(r_{2}\right)=0, \mathrm{u}_{0}(\delta)=\mathrm{V}, \mathrm{u}_{1}\left(\mathrm{r}_{2}\right)=0 \\
& u_{1}(\delta)=-\frac{\partial u_{0}(\delta)}{\partial r}
\end{aligned}
$$

The solutions of (16-17) with the boundary are easily found to be

$$
\begin{aligned}
u_{0}(z, t)= & \frac{1}{4} \frac{d p}{d z}\left(r^{2}-r_{2}^{2}\right) \\
& +\frac{V-\frac{1}{4} \frac{d p}{d z}\left(\delta^{2}-r_{2}^{2}\right)}{\log \left[\frac{\delta}{r_{2}}\right]} \log \left[\frac{r}{r_{2}}\right] \\
u_{1}(z, t)= & \frac{\frac{\delta^{2}}{2} \frac{d p}{d z}+\frac{V-\frac{1}{4} \frac{d p}{d z}\left(\delta^{2}-r_{2}^{2}\right)}{\log \left[\frac{\delta}{r_{2}}\right]}}{\left(\frac{\delta^{2}}{r_{2}^{2}}-1\right)} \\
& \left(\frac{1}{r}-\frac{r}{r_{2}^{2}}\right) .
\end{aligned}
$$

Following the analysis given by Mekheimer [7], the mean volume flow, $\bar{Q}$ over a period is obtained as

$$
\begin{aligned}
Q(z, t)= & \frac{\bar{Q}}{\pi}-\frac{\phi^{2}}{2}+2 \phi \operatorname{Cos}[2 \pi(\mathrm{z}-\mathrm{t})] \\
& +\phi^{2} \operatorname{Cos}[2 \pi(\mathrm{z}-\mathrm{t})]^{2}
\end{aligned}
$$

Where $\bar{Q}$ is the time-average of the flow over one period of the wave.

Make combination between Equations(2.17 and 2.18) we get

$$
\begin{gathered}
\frac{\partial p}{\partial z}=\frac{S}{C} \\
S=\frac{\frac{\bar{Q}}{\pi}-\frac{\phi^{2}}{2}+2 \phi \operatorname{Cos}[2 \pi(-\mathrm{t}+\mathrm{z})]+\phi^{2} \operatorname{Cos}[2 \pi(-\mathrm{t}+\mathrm{z})]^{2}}{2 \pi} \\
-\frac{V\left(0.25 r_{1}^{2}-0.5 \log \left[r_{1}\right] r_{1}^{2}-\frac{r_{2}^{2}}{4}+\frac{1}{2} \log \left[r_{2}\right] r_{2}^{2}\right)}{\log [\delta]} \\
-\frac{V \epsilon \operatorname{Cos}[\theta]\left(-\mathrm{r}_{1}+\frac{\mathrm{r}_{1}^{3}}{3}+\mathrm{r}_{2}-\frac{\mathrm{r}_{2}^{3}}{3}\right)}{\left(-1+\delta^{2}\right) \log [\delta]}
\end{gathered}
$$




$$
\begin{aligned}
C= & -\frac{r_{1}^{4}}{16}+\frac{r_{2}^{4}}{16}+\frac{1}{8}\left(r_{1}^{2}-r_{2}^{2}\right)+\frac{0.25\left(1-\delta^{2}\right)\left(0.25 r_{1}^{2}-0.5 \log \left[r_{1}\right] r_{1}^{2}-\frac{r_{2}^{2}}{4}+\frac{1}{2} \log \left[r_{2}\right] r_{2}^{2}\right)}{\log [\delta]} \\
& +\frac{0.5 \delta^{2} \epsilon \operatorname{Cos}[\theta]\left(-\mathrm{r}_{1}+\frac{\mathrm{r}_{1}^{3}}{3}+\mathrm{r}_{2}-\frac{\mathrm{r}_{2}^{3}}{3}\right)}{-1+\delta^{2}}+\frac{0.25\left(1-\delta^{2}\right) \epsilon \operatorname{Cos}[\theta]\left(-\mathrm{r}_{1}+\frac{\mathrm{r}_{1}^{3}}{3}+\mathrm{r}_{2}-\frac{\mathrm{r}_{2}^{3}}{3}\right)}{\left(-1+\delta^{2}\right) \log [\delta]}
\end{aligned}
$$

where $\mathrm{S}, \mathrm{C}$ are the numerator and dominator of pressure gradient.

The pressure rise $\Delta p(t)$ and the friction force (at the wall) on the outer and inner tubes are $F_{2}(t)$ and $F_{1}(t)$ respectively, in a cylinder of length $\mathrm{L}$, in their non-dimensional forms, are given by

$$
\begin{aligned}
& \Delta p(t)=\int_{0}^{1} \frac{\partial p}{\partial z} d z \\
& F_{1}(t)=\int_{0}^{1} r_{1}^{2}\left(-\frac{\partial p}{\partial z}\right) d z \\
& F_{2}(t)=\int_{0}^{1} r_{2}^{2}\left(-\frac{\partial p}{\partial z}\right) d z
\end{aligned}
$$

The average rise in pressure is $\overline{\Delta p}$, outer and inner frictional forces $\overline{F_{2}}, \overline{F_{1}}$ are then evaluated by averaging $\Delta p(t), F_{1}(t), F_{2}(t)$ over one period of the wave.

By substituting in Equations(2.20-2.22) with $r_{1}=\delta+$ $\epsilon \operatorname{Cos}[\theta], r_{2}=1+\phi \operatorname{Cos}[2 \pi(\mathrm{z}-\mathrm{t})]$, we get the average pressure rise and the average friction force (at the wall) on the outer and inner tubes.

\section{Graphical results and discussion}

The numerical evaluations of the analytic results obtained for the pressure gradient $\frac{d p}{d z}$, the pressure rise $\Delta p(t)$, the frication force (at the wall) $F_{1}(t), F_{2}(t)$, on the inner and outer tubes and the basic flow velocity $u(r, t)$ for various values of the dimensionless time $t$, flow rate $Q$, amplitude ratio $\phi$, the velocity of the inner tube $V$, and the eccentricity parameter $\epsilon$ are discussed.

Also, the effects of pertinent parameters on the average pressure $\Delta \bar{p}$ and frictional force $\bar{F}_{1}, \bar{F}_{2}$ per one wave length are investigated by numerical integrations.

In Figs. 2-3, the variation of $\frac{d p}{d x}$ versus $\mathrm{z}$ is shown for different values of $\epsilon, \phi, \bar{Q}$ and $\delta$ by fixing the other parameters. We can see that, the pressure gradient is relatively small in wider part of the channel, that is, the flow can easily pass without imposition of large pressure gradient. Where, in a narrow part of the channel, a much larger pressure gradient maintain the same flux to pass it, especially for is required to the narrowest position near $\mathrm{x}=0.5$. This is in well agreement with the physical situation.

Also, it is observed that the maximum amplitude of the pressure gradient increases as the eccentricity parameter $\epsilon$, the radius of the inner tube $\delta$ and $\phi$ increases, while it's

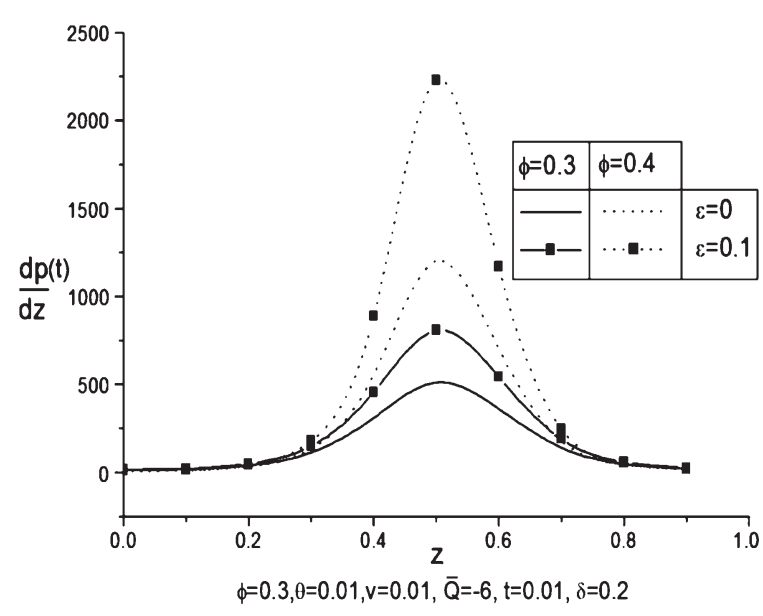

Fig. 2. Variation of pressure gradient over the length versus $z$ with different values of amplitude ratio $\phi$ with different $\epsilon$.

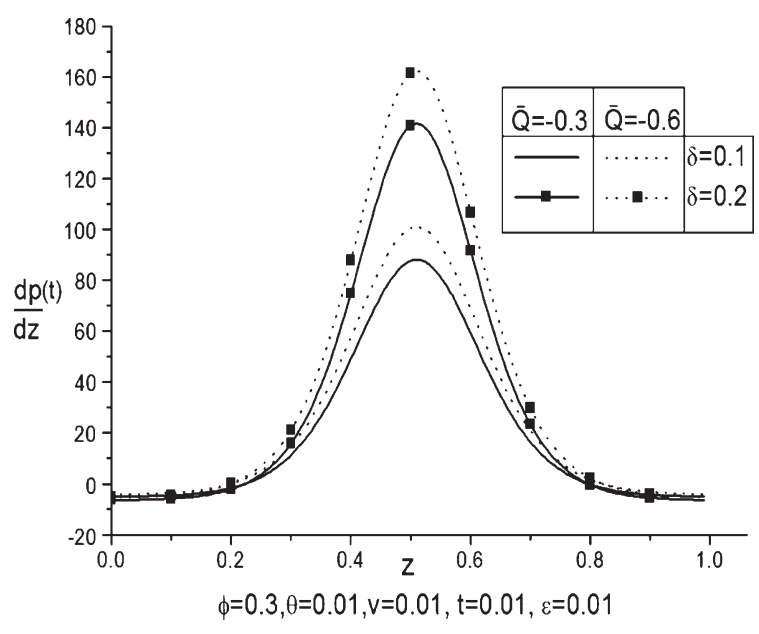

Fig. 3. Variation of pressure gradient over the length versus $z$ with different values of flow rate $\bar{Q}$ with different $\delta$. 


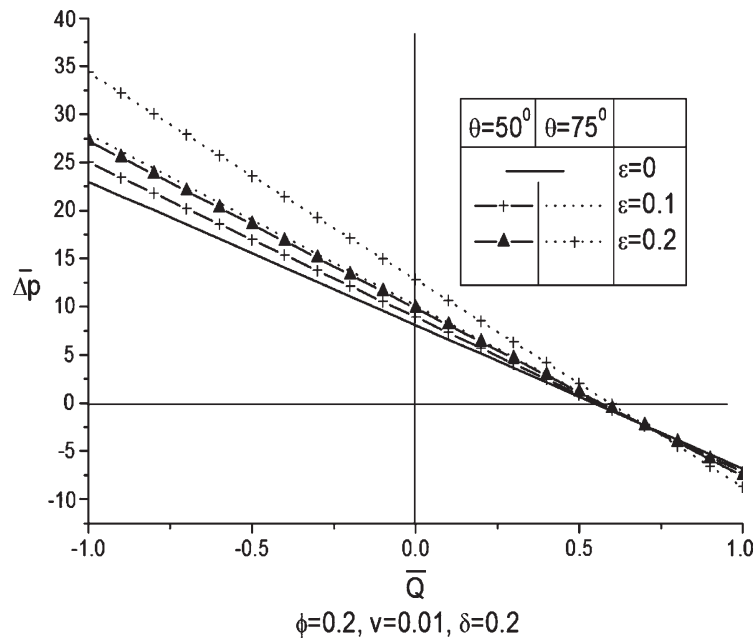

Fig. 4. Variation of pressure rise over the length versus $\bar{Q}$ with different values of eccentric parameter $\epsilon$ with different $\theta$.

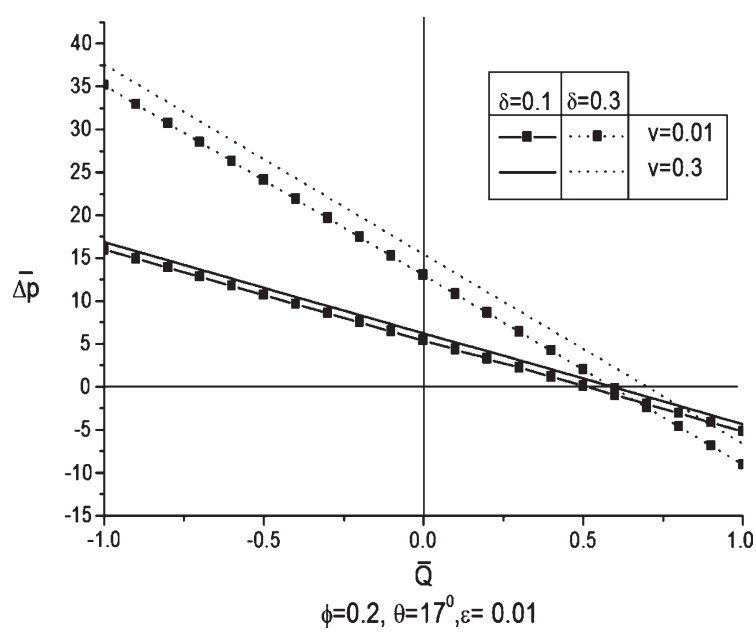

Fig. 5. Variation of pressure rise over the length versus $\bar{Q}$ with different values of inner cylinder velocity $v$ with different $\delta$.

decreases as the flow rate $Q$ increases. Hence, the pressure gradient is higher for the eccentric flow than that for the concentric one $(\epsilon=0)$, and for a small radius of the inner tube we get a low pressure gradient in the gap of the annulus and this alternately achieve the flow rate. Figures 4 and 5 illustrate the change of the average pressure rise $\Delta \bar{p}$ versus the time-averaged mean flow rate $\bar{Q}$ for various values of the $\theta, \epsilon, \delta$ and $V$. The graph illustrate the pumping regions, which are, the peristaltic pumping $(\bar{Q}>0, \Delta \bar{p}>0)$, the augmented pumping $(\bar{Q}>0, \Delta \bar{p}<0)$ and the retrograde pumping $(\bar{Q}<0, \Delta \bar{p}>0)$. These figures shows that that there is an inversely linear relation between $\Delta \bar{p}$ and $\bar{Q}$, that is, the pressure rise decreases with increasing the flow rate and the pumping curves are linear both for eccentric and

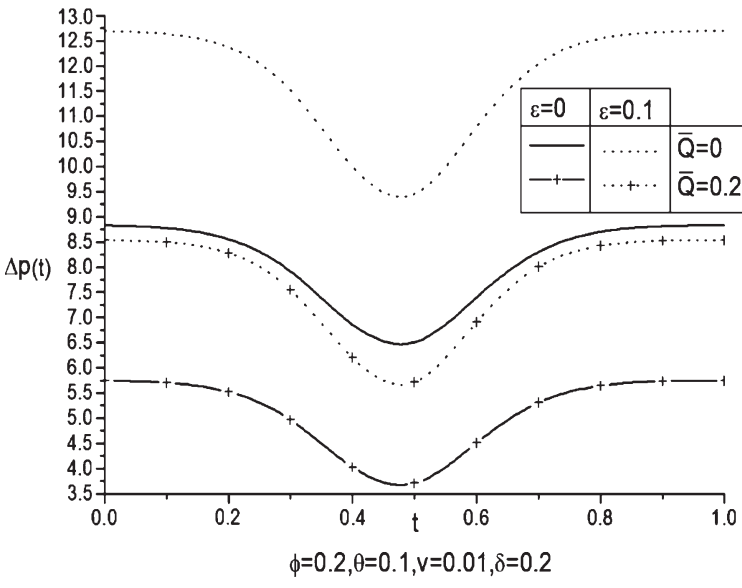

Fig. 6. Variation of pressure rise over the time interval $t$ with different values of eccentric parameter $\epsilon$ with different main flow rate values.

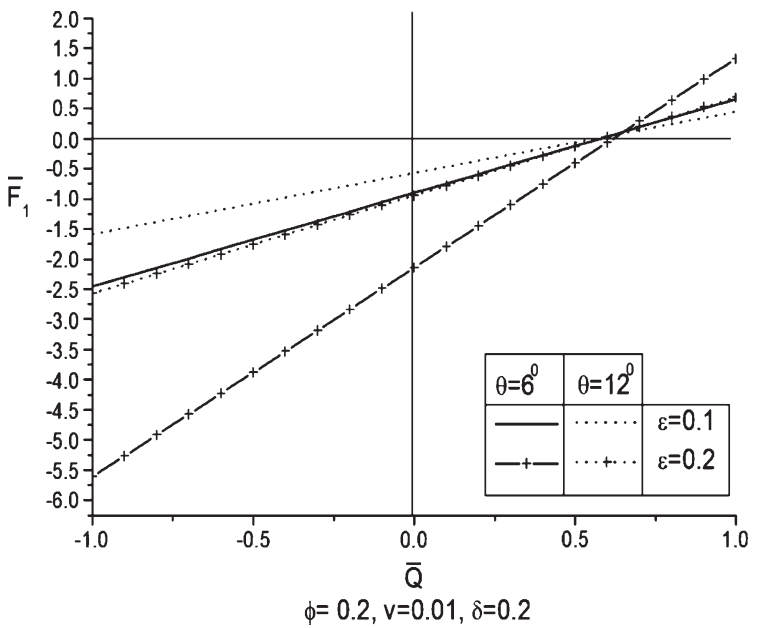

Fig. 7. Variation of the average inner Friction force over length versus $\bar{Q}$ with different values of eccentric parameter $\epsilon$ at different $\theta$.

concentric flow. Moreover, the pumping curves for eccentric flow lie above the curves of the concentric flow in pumping region $(\Delta \bar{p}>0)$. Also, the pressure rise increases as the eccentricity parameter $\epsilon$ and the angle $\theta$ increases, and for fixed value $(\Delta \bar{p})$, the flow rate will increases as $\epsilon$ and $\theta$ increases, i.e, the back flow decreases. Figure 5. shows that, as the velocity of the inner tube $V$ or it's radius $\delta$ increase, the average pressure rise will increases in the backward and peristaltic pumping region, while it decreases in the augmented pumping region. Also, for fixed $(\Delta \bar{p})$ the flow rate will increase as $V$ and $\delta$ increases.

Figure 6. illustrate the variation of the pressure rise with time which indicates that $\Delta p(t)$ is heigher for the eccentric cylinder than that for concentric one, and this pressure 


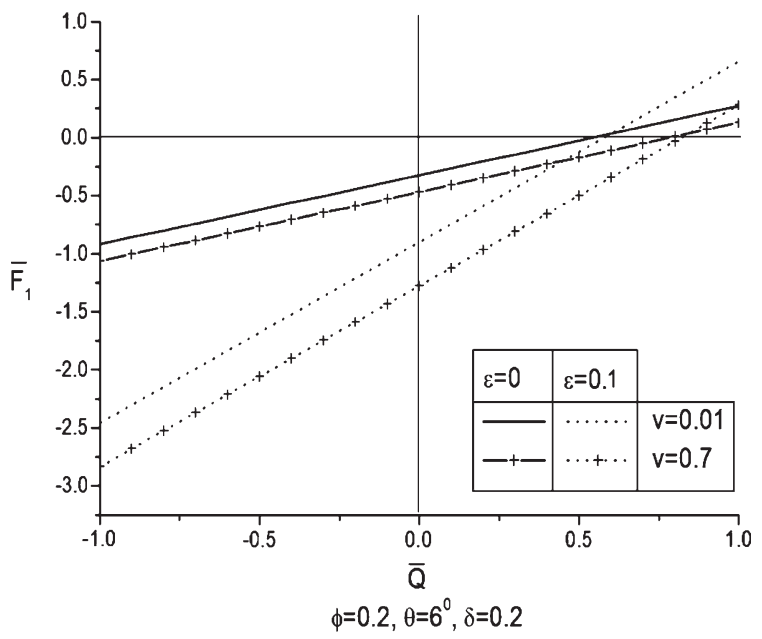

Fig. 8. Variation of the average inner Friction force versus $\bar{Q}$ with different values of $\epsilon$ and $v$.

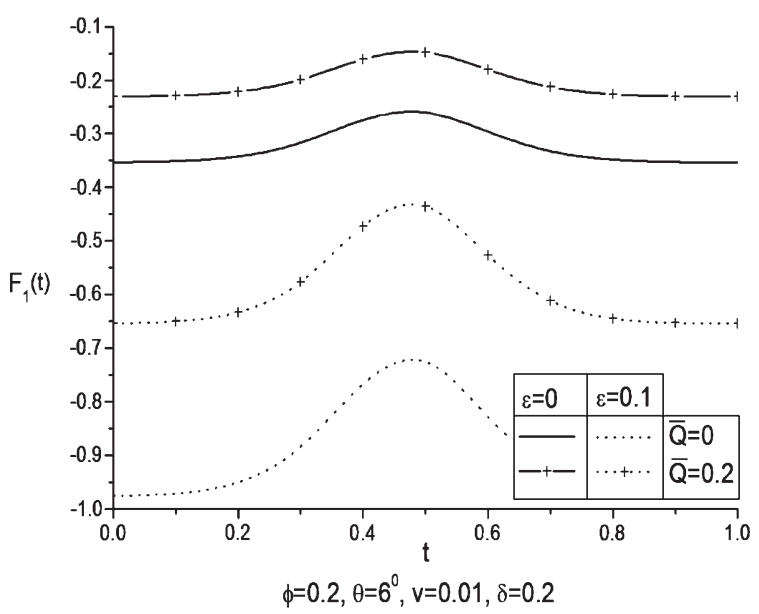

Fig. 9. Variation of inner Friction force versus $t$ with different values of the main flow rate $Q$.

will increases as the eccentricity parameter $\epsilon$ increases. Also, $\Delta p(t)$ will decrease as $\mathrm{Q}$ increases.

The inner friction force ( on the inner tube) as well as the outer friction force ( on the outer tube) are plotted in Figs. 7-12 versus the dimensionless time $t$ and the flow rate $Q$. We notice from these figures that the inner and outer friction forces have the opposite behavior compared to the pressure rise. The inner friction force behaves similar to the outer friction force for the same values of the parameters, moreover the outer friction force is greater than the inner friction force at the same values of the parameters. Furthermore, from Figs. 9 and 12 it is noticed that the inner and outer friction forces are higher for concentric cylinder than that for the eccentric one. Figures 13-16 describes the variation of the basic flow with

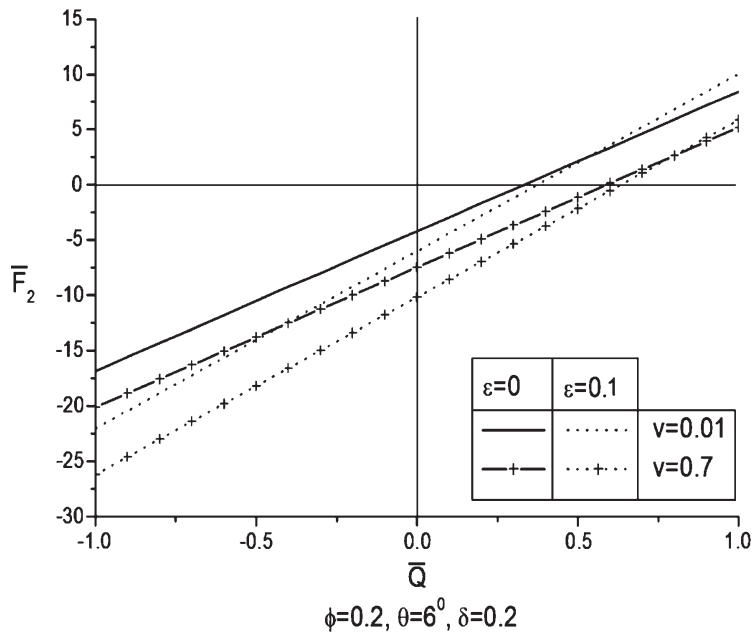

Fig. 10. Variation of the average outer Friction force versus $\bar{Q}$ for different values of $v$ at different values of $\epsilon$.

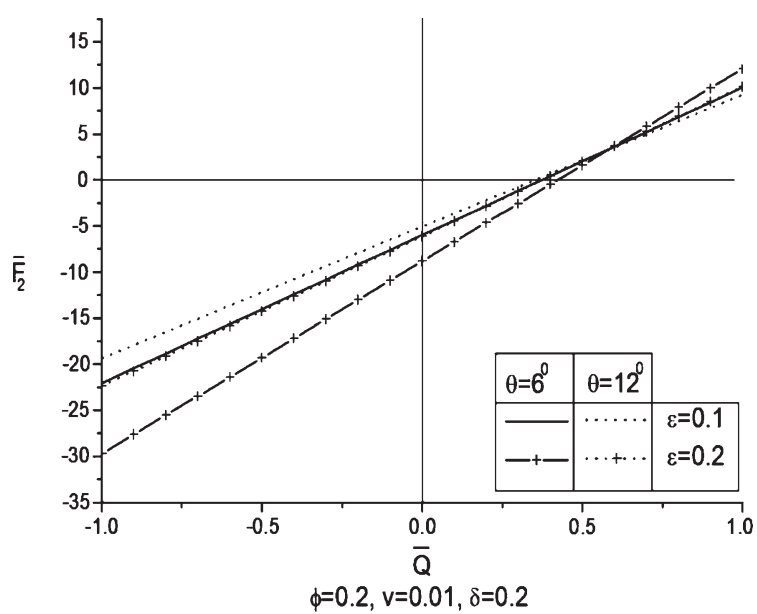

Fig. 11. Variation of outer Friction force versus $\bar{Q}$ for different values of $v$ at different values of $\epsilon$.

$\mathrm{r}$ and $\mathrm{t}$ for different values of different governing parameters. In Fig. 13 it is observed that, the basic flow increase as the eccentric parameter $\epsilon$ and the flow rate $Q$ increases.

Also, as expected at $\epsilon=0$ the velocity value of the basic flow started at the radius of the inner tube, where $\delta=0.1$ and ends at $r=1$. Hence, the basic flow is higher for eccentric tube than that for concentric one. Figure 14. depicts the variation of the basic flow with $r$ for different values of the amplitude ratio and t. We see that, as $\phi$ (the amplitude ratio of the peristaltic wave) increases the basic flow velocity increases, while it's decreases with time. For the effect of the inner tube radius $\delta$, the basic flow is slow near the region of the inner tube and as $\delta$ increases the basic flow manages to overtake the latter. Figures 15-16. indicates that 


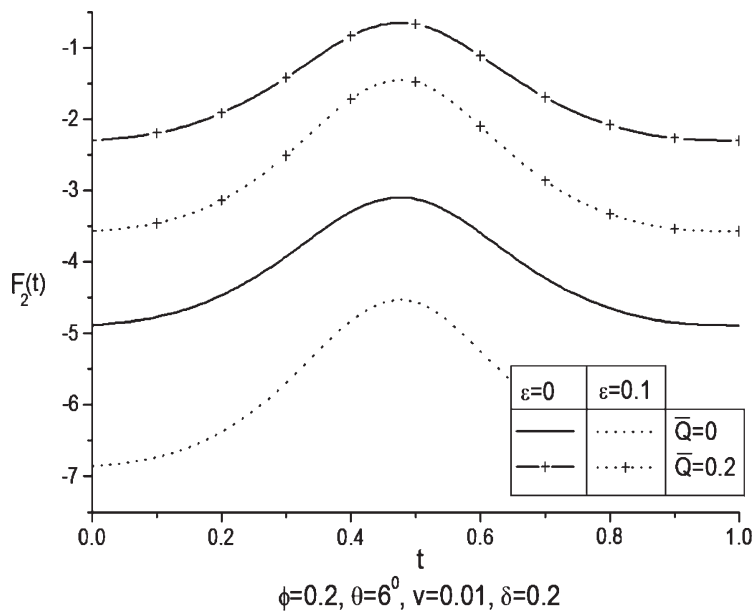

Fig. 12. Variation of outer Friction force versus $t$ with different values $\epsilon$.

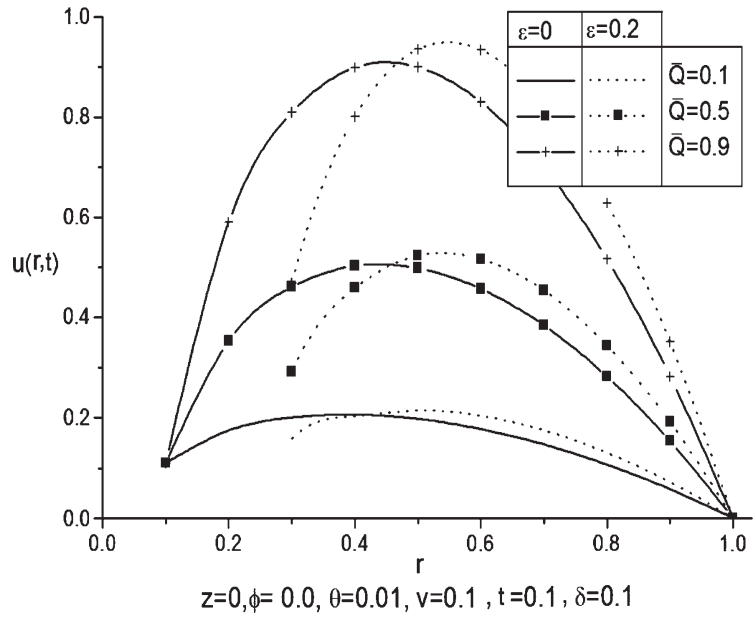

Fig. 13. The basic thread-annular flow $u(r, t)$ for various $\bar{Q}$.

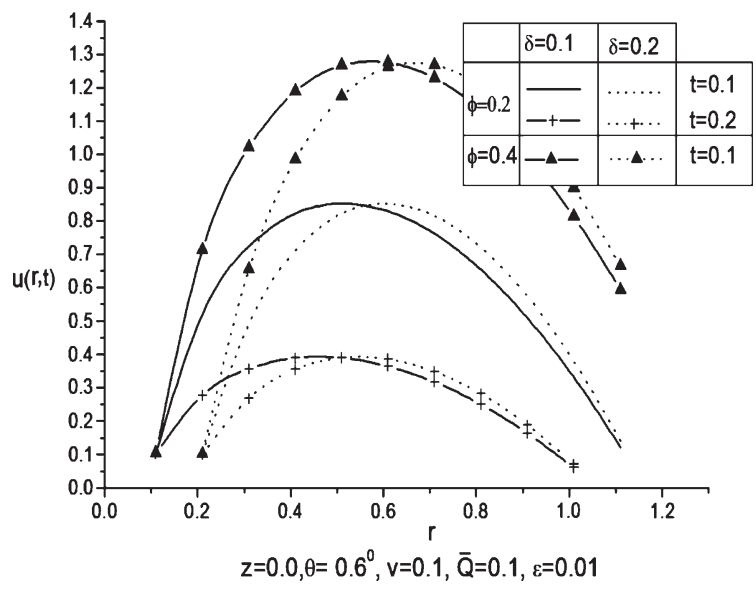

Fig. 14. $u(r, t)$ for various $\delta$ with $t$ at different state of $\phi$.

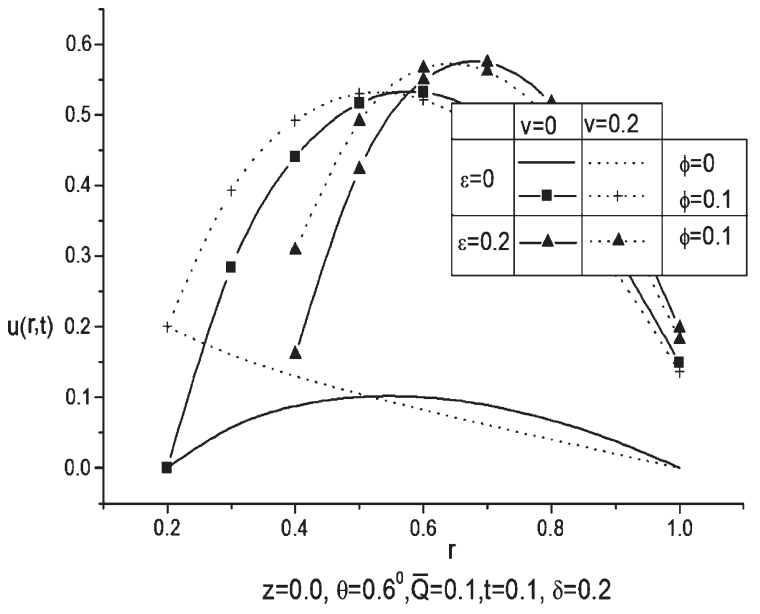

Fig. 15. The basic thread-annular flow $u(r, t)$ for various $V$.

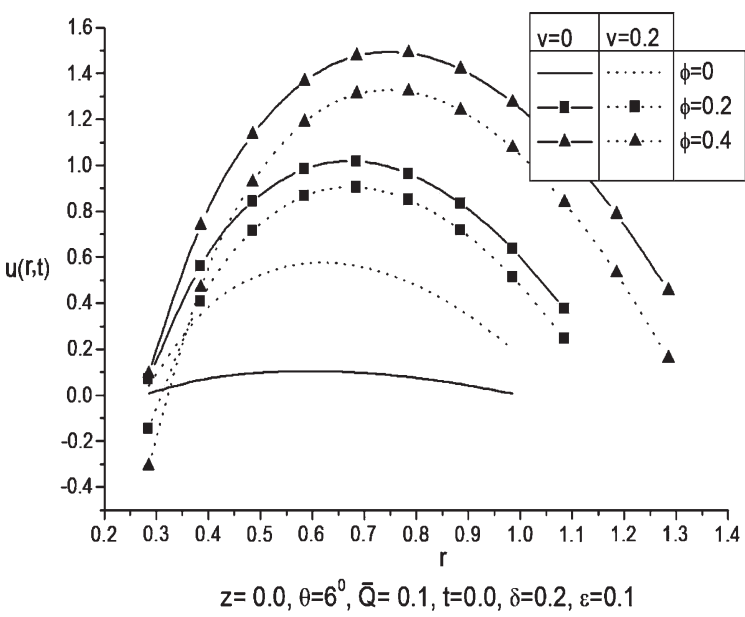

Fig. 16. The basic thread-annular flow $u(r, t)$ for various $V$.

the basic wq1flow will increase as the velocity of the inner tube increase for $\phi=0$ ( no peristaltic wave), but for $\phi \neq 0$ this effect is reverse.

\section{References}

[1] S. Nadeem and N.S. Akbar, Influence of heat and mass transfer on a peristaltic motion of a Jeffrey-six constant fluid in an annulus, Heat and Mass Transfer 46 (2010), 485-493.

[2] Kh.S. Mekheimer and A.N. Abdel-Wahab, Compressibility effects on peristaltic flow of a non-Newtonian Maxwell fluid through an annulus, chapter 7 In the Book : Fluid Transport: Theory, Dynamics and Transport, Editor: Emma T. Berg, ISBN: 978-1-61122-317-0, c: 2010 Nova Science Publishers, Inc.

[3] S. Nadeem and N.S. Akbar, Effects of heat and mass transfer on peristaltic flow of Carreau fluid in a vertical annulus, Zeitschrift fur Naturforschung 65a (2010), 1-12. 
[4] S. Nadeem and N.S. Akbar, Influence of radially varying MHD on the peristaltic flow in an annulus with heat and mass transfer, Taiwan Institute of Chemical Engineers 41 (2010), 286-294.

[5] S. Nadeem, N.S. Akbar and K. Vajravelu, Peristaltic flow of a Sisko fluid in an endoscope: Analytical and numerical solutions, International Journal of Computer Mathematics $\mathbf{8 8}$ (2011), 1013-1023.

[6] Kh.S. Mekheimer and Y. Abdelmaboud, Peristaltic flow of a couple stress fluid in an annulus: Application of an endoscope, Physica A 387 (2008), 2403-2415.

[7] Kh.S. Mekheimer, Peristaltic transport of a Newtonian fluid through a uniform and non-uniform annulus, The Arabian $J$ for Sc and Eng 30(1A) (2005), 1-15.

[8] Abd El Hakeem Abd El Naby, A.E.M. El Misery and I.I. El Shamy, Effects of an endoscope and fluid with variable viscosity on peristaltic motion, Applied Mathematics and Computation 158 (2004), 497-511.

[9] Y. Abd Elmaboud, Influence of induced magnetic field on peristaltic flow in an annulus, Communications in Nonlinear Science and Numerical Simulations 17(2) (2011), 685-698.

[10] Kh.S. Mekheimer and Y. Abd elmaboud, Peristaltic transport of a particle-fluid suspension through a uniform andnonuniform annulus, J Applied Bionicand Biomech 5(2) (2008), 47-57.

[11] Kh.S. Mekheimer and Y. Abd elmaboud, The influences ofa micropolar fluid on peristaltic transport in anannulus:
Application of a clot model, J Applied Bionic and Biomech 5(1) (2008), 13-23.

[12] Kh.S. Mekheimer and A.N. Abdel-Wahab, Net annulus flow of a compressible viscous Liquid with peristalsis, Journal of Aerospace Engineering 25(4) (2012), 660-669.

[13] Kh.S. Mekheimer and Y. Abd elmaboud, Peristaltic Flow through a Porous Medium in an Annulus: Application of an Endoscope, Applied Mathematics and Information Sciences, 2(1) (2008), 103-111.

[14] J.M. Nouri, H. Umur and J.H. Whitelaw, Flow of Newtonian and non-Newtonian fluids in concentric and eccentric annuli, J Fluid Mech 253 (1993), 617-641.

[15] I.C. Walton and S.H. Bittleston, The axial flow of a Bingham plastic in a narrow eccentric annulus, J Fluid Mech 222 (1991), 39-60.

[16] Irene Dris and Eric S.G. Shaqfeh, Flow of aviscoelastic fluid between eccentric cylinders: Impact on flow stability, J NonNewtonian Fluid Mech 80 (1998), 59-78.

[17] C.H. Frei, P. Luscher and E. Wintermantel, Thread-annular flow in vertical pipes, Journal of Fluid Mechanics 410 (2000), 185-210.

[18] J. Labadin and A.G. Walton, Modeling of axial flow between eccentric cylinders, Proceedings of the 2nd IMT-GT Regional Conference on Mathematics, Statistics and Applications Universiti Sains Malaysia, Penang, June 13-15, 2006. 

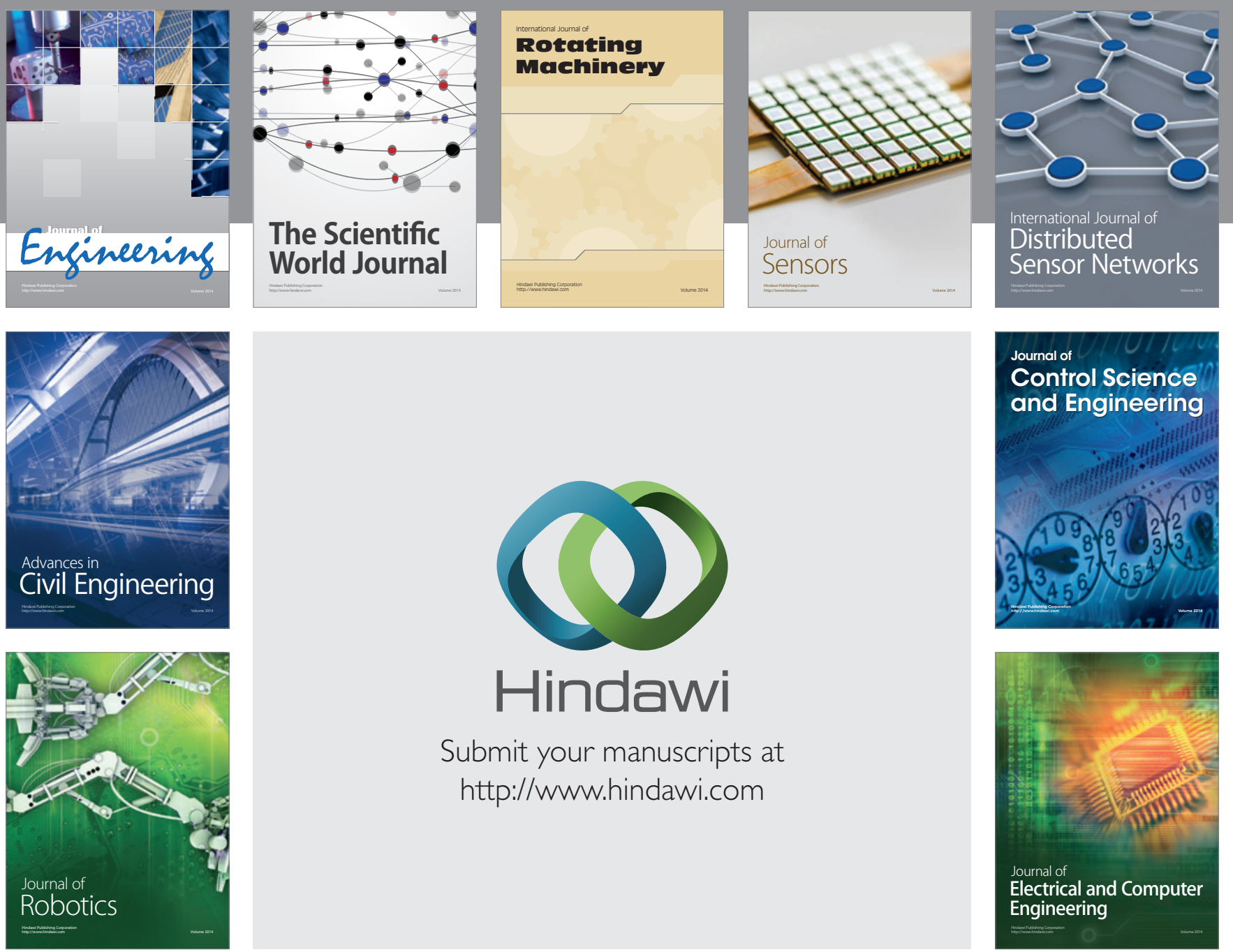

Submit your manuscripts at

http://www.hindawi.com
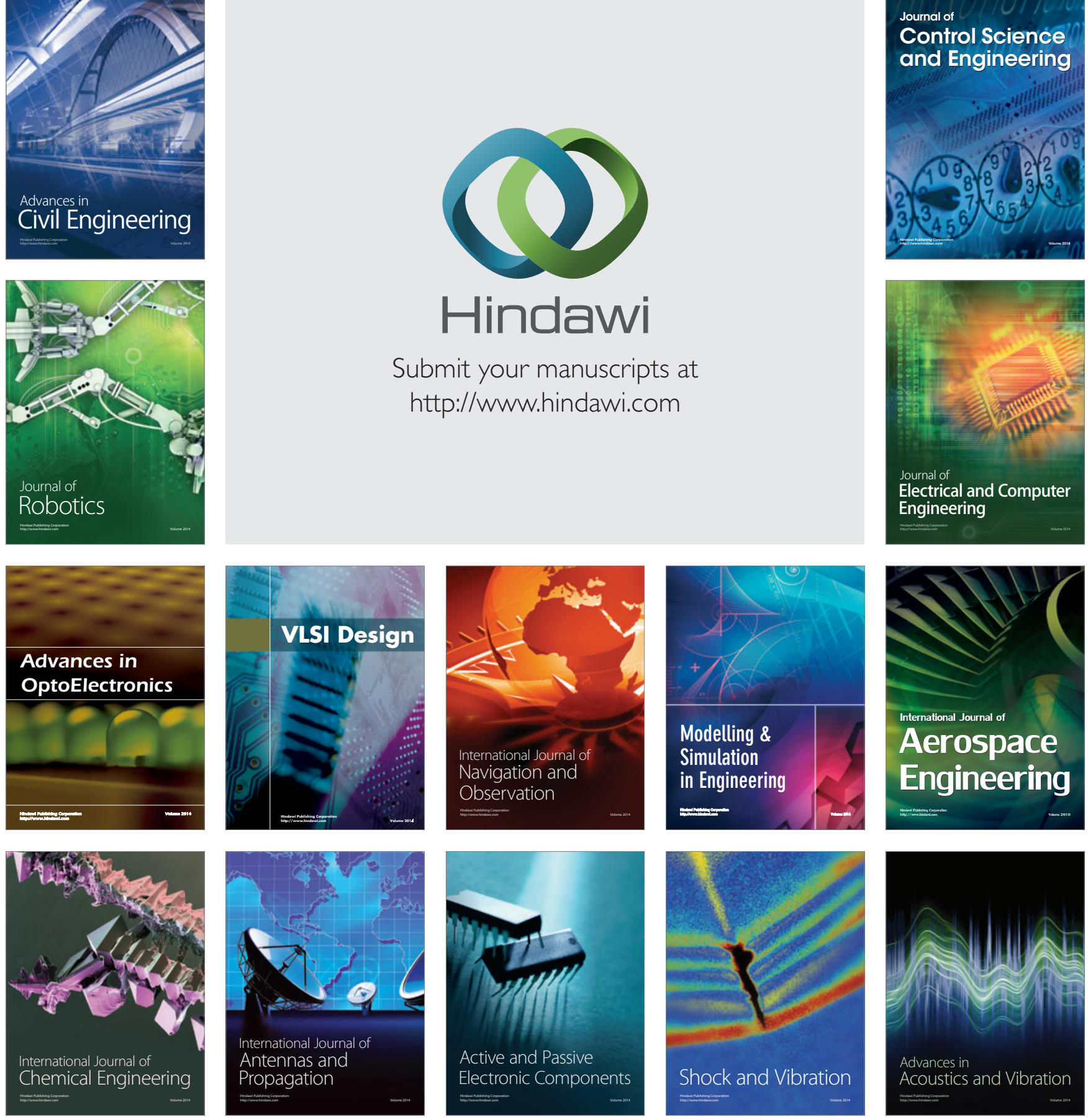